\title{
ASCOLI THEOREMS AND THE EXPONENTIAL MAP
}

\author{
BY \\ N. NOBLE $\left({ }^{1}\right)$
}

Introduction. Let $Y$ and $Z$ be topological spaces and let $H$ be a subset of $F(Y, Z)$, the set of all functions from $Y$ to $Z$. We say that $H$ is pointwise bounded if $\{f(y): f \in H\}$ has compact closure in $Z$ for each point $y$ in $Y$. Following [25] we say that $H$ is evenly continuous if, for each $y$ in $Y$, each $z$ in $Z$, and each neighborhood $U$ of $z$ there exists neighborhoods $V$ of $y$ and $W$ of $z$ such that $f(V) \subseteq U$ whenever $f$ is in $H$ and $f(y)$ is in $W$. If $\tau$ is a topology on $C(Y, Z)$, the set of continuous functions in $F(Y, Z)$, then $(Y, Z, \tau)$ is said to satisfy the weak Ascoli theorem if each $\tau$-closed, pointwise bounded, evenly continuous subset of $C(Y, Z)$ is $\tau$-compact. If also each $\tau$-compact subset is $\tau$-closed, pointwise bounded and evenly continuous, then $(Y, Z, \tau)$ is said to satisfy the Ascoli theorem.

The prototype of the Ascoli theorems was proved by Ascoli in [4] and independently by Arzelá, who acknowledged Ascoli's priority in [3]. Since then a host of results which may justifiably be called Ascoli theorems have been discovered, many of which do not satisfy our definition of the Ascoli, or a weak Ascoli theorem. (Some, such as Theorem 3-37 of [23], and Theorem 2 of [10] are stated for sets of functions other than $C(Y, Z)$, and in some, such as Theorem 7-18 of [25], even continuity is replaced by a nonequivalent condition.) Some of the "Ascoli theorems" of this type will be considered in $\S 5$.

Our approach is established in the next section where we prove, under slight restrictions on $Z$ and $\tau$, the equivalence of the statements " $(Y, Z, \tau)$ satisfies a weak Ascoli theorem" and " $(Y, Z, \tau)$ satisfies an Ascoli theorem" with two statements involving the exponential map. The second and third sections are devoted to a study of the exponential map, and in the fourth section we apply our results to give a number of Ascoli theorems. The final section contains a discussion of several ways in which our results and techniques can be generalized.

1. The equivalent statements. Let $e: F(Y, Z) \times Y \rightarrow Z$ be the map which takes $(f, y)$ to $f(y)$ and let $\mu: F(X \times Y, Z) \rightarrow F(X, F(Y, Z))$ be the map defined by the relations: $\mu f(x)(y)=f(x, y)$. The restrictions of these maps to subspaces will also be denoted by $e$ and $\mu$ respectively; $e$ is called the evaluation map and $\mu$ the exponential map. When, $\tau$ is a topology for $C(Y, Z)$ we say that $(X, Y, Z, \tau)$

Presented to the Society, January 27, 1967 under the title Necessary and sufficient conditions that Ascoli's theorem hold; received by the editors December 16, 1968.

( $\left.{ }^{1}\right)$ This paper is an expanded version of the second chapter of the author's doctoral dissertation, which was written at the University of Rochester under the direction of Professor W. W. Comfort. Work on this paper was partially supported by NASA grant number 93-6. 
satisfies the partial exponential law if $\mu(C(X \times Y, Z)) \subseteq C\left(X, C(Y, Z)_{\tau}\right)$. If equality holds, $(X, Y, Z, \tau)$ is said to satisfy the exponential law. (The term "exponential law" derives from the fact that, with different notation and with $\tau$ and $\mu$ understood, this equality reads: $(Z)^{X \times Y}=\left(Z^{Y}\right)^{X}$.)

Let $\tau_{p}$ denote the topology of pointwise convergence on $F(Y, Z)$ and its subspaces, and recall that $\tau_{p}$ is Hausdorff if and only if $Z$ is Hausdorff. It is obvious that $\mu^{-1}\left(C\left(X, C(Y, Z)_{\tau_{p}}\right)\right)$ is just the set of separately continuous functions on $X \times Y$, for arbitrary spaces $X, Y$, and $Z$, and hence that $\mu(C(X \times Y, Z))$ is always contained in $C\left(X, C(Y, Z)_{\tau_{p}}\right)$. The following lemma is an immediate consequence of Theorems 7-19 and 7-20 of [25].

1.1 Lemma. Let $Z$ be regular, $Y$ arbitrary, and let $H$ be a subset of $F(Y, Z)$. If $H$ is $\tau_{p}$-compact then $H$ is evenly continuous if and only if the evaluation map $e$ on $H \times Y$ is continuous when $H$ has the topology $\tau_{p}$. If $H$ is evenly continuous then the $\tau_{p}$-closure of $H$ in $F(Y, Z)$ is also evenly continuous, and is therefore contained in $C(Y, Z)$.

1.2 THEOREM. Let $Z$ be regular Hausdorff, $Y$ arbitrary, and $\tau$ a topology for $C(Y, Z)$ such that $\tau \geqq \tau_{p}$. Then $(Y, Z, \tau)$ satisfies the weak Ascoli theorem if and only if, for each compact Hausdorff space $K,(K, Y, Z, \tau)$ satisfies the partial exponential law.

Proof. First suppose $(Y, Z, \tau)$ satisfies the weak Ascoli theorem. Let $K$ be compact Hausdorff and let $f \in C(K \times Y, Z)$. Then $\mu f: K \rightarrow C(Y, Z)_{\tau_{p}}$ is continuous, so $\mu f(K)$ is $\tau_{p}$-compact and hence pointwise bounded and (since $\tau \geqq \tau_{p}$ ) $\tau$-closed. It suffices to show that $\mu f(K)$ is evenly continuous, since then it follows that $\mu f(K)$ is $\tau$-compact so $\tau$ and $\tau_{p}$ coincide on $\mu f(K)$ and hence $\mu f: K \rightarrow C(Y, Z)_{\tau}$ is continuous. Let $y \in Y, z \in Z$ and let $U$ be an open neighborhood of $z$. Let $W$ be a closed neighborhood of $z$ with $W \subseteq U$ and let

$$
K^{\prime}=\left\{x \in K:(x, y) \in f^{-1}(W)\right\} ;
$$

since $f^{-1}(W)$ is closed, $K^{\prime}$ is compact and hence the projection $\pi_{Y}: K^{\prime} \times Y \rightarrow Y$ is a closed map. Thus $V=Y \mid \pi_{Y}\left(K^{\prime} \times Y \backslash f^{-1}(U)\right)$ is open, and since $K^{\prime} \times\{y\}$ $\subseteq f^{-1}(W) \subseteq f^{-1}(U), y$ is in $V$. Now if $g \in \mu f(K)$ and $g(y) \in W$, then $g$ is in $\mu f\left(K^{\prime}\right)$; so $g(V) \leqq U$. Thus $K$ is evenly continuous.

Now suppose that $(K, Y, Z, \tau)$ satisfies the partial exponential law for each compact Hausdorff space $K$, and let $K^{\prime} \subseteq C(Y, Z)$ be $\tau$-closed, pointwise bounded and evenly continuous. Then by Lemma $1.1, K$, the $\tau_{p}$-closure of $K^{\prime}$ in $F(Y, Z)$, is evenly continuous, so $K \subseteq C(Y, Z)$ and $e: K \times Y \rightarrow Z$ is continuous. Since $K$ is compact, $\mu e: K \rightarrow C(Y, Z)_{\tau}$ is continuous. But $\mu e$ is just the inclusion, so $K$ is $\tau$-compact. Since $K^{\prime}$ is a closed subset of $K$, it follows that $K^{\prime}$ is $\tau$-compact.

1.3 THEOREM. Let $Z$ be regular Hausdorff, $Y$ arbitrary, and $\tau$ a topology for $C(Y, Z)$ such that $\tau \geqq \tau_{p}$. Then $(Y, Z, \tau)$ satisfies the Ascoli theorem if and only if for each compact Hausdorff space $K,(K, Y, Z, \tau)$ satisfies the exponential law. 
Proof. If $(Y, Z, \tau)$ satisfies the Ascoli theorem, if $K$ is compact, and if $f \in$ $F(K \times Y, Z)$ is such that $\mu f: K \rightarrow C(Y, Z)_{\tau}$ is continuous, then $e: K \times Y \rightarrow Z$ is continuous (with respect to $\tau_{p}$ which coincides with $\tau$ on the compact subset $\mu f(K))$ so $f=e \circ(\mu f \times 1)$ is continuous. Conversely, if $K \subseteq C(Y, Z)$ is $\tau$-compact and $(K, Y, Z, \tau)$ satisfies the exponential law, then since the inclusion $i: K \rightarrow C(Y, Z)$ is continuous, $\mu^{-1}(i)=e$ is continuous so by Lemma $1.1 \mathrm{~K}$ is evenly continuous. The remaining implications are Theorem 1.2.

Note that in the proof above we have actually established a slightly stronger result, viz., that each $\tau$-compact subset is evenly continuous if and only if for each compact $K, C(K \times Y, Z) \supseteq \mu^{-1}\left(C\left(K, C(Y, Z)_{\tau}\right)\right)$. Also, the requirement that $\tau$ be greater than or equal to $\tau_{p}$ in the hypotheses of the two theorems above can be weakened. The condition which is actually used is that $\tau$ is Hausdorff and coincides with $\tau_{p}$ on each $\tau$-compact evenly continuous subset of $C(Y, Z)$. By Exercise 7-C of [25], the topology of pointwise convergence on a dense subset of $Y$ (which can be strictly smaller than $\tau_{p}$ ) satisfies these conditions when $Z$ is completely regular Hausdorff.

If $Z^{\prime}$ is a subspace of $Z$ then, under the natural identification, $C\left(Y, Z^{\prime}\right)$ is a subset of $C(Y, Z)$ and $C\left(X \times Y, Z^{\prime}\right)$ is a subset of $C(X \times Y, Z)$. Since $f(X \times Y)$ is contained in $Z^{\prime}$ if and only if $\mu f(X)(Y)$ is contained in $Z^{\prime}$, we have

1.4 COROLlaRY. Let $Z$ be regular Hausdorff, $Y$ arbitrary, $\tau$ a topology on $C(Y, Z)$ such that $\tau \geqq \tau_{p}$, let $Z^{\prime}$ be a subspace of $Z$, and let $\tau^{\prime}$ denote the relative topology on $C\left(Y, Z^{\prime}\right)$. If $(Y, Z, \tau)$ satisfies the Ascoli theorem or the weak Ascoli theorem, then so does $\left(Y, Z^{\prime}, \tau^{\prime}\right)$.

When $\tau$ is one of the "usual" topologies for $C(Y, Z), \tau^{\prime}$ will often be the related " usual" topology for $C\left(Y, Z^{\prime}\right)$ : for instance, when $\tau$ is the compact-open topology on $C(Y, Z), \tau^{\prime}$ is the compact-open topology on $C\left(Y, Z^{\prime}\right)$. Note that the conditions " $H \subseteq C(Y, Z)$ is pointwise bounded" and " $H \subseteq C(Y, Z$ ') is pointwise bounded" are not equivalent. An interesting consequence of this fact and the corollary is: For $Z, Z^{\prime}$, and $\tau$ as above, if $(Y, Z, \tau)$ satisfies the Ascoli theorem, then $C\left(Y, Z^{\prime}\right)$ is $k$-closed (meets each compact set in a closed set) in $C(Y, Z)_{\tau}$ if and only if $Z^{\prime}$ is $k$-closed in $Z$.

2. The partial exponential laws. In order to apply the results of the preceding section we need some additional information about the exponential map which will be provided here and in $\$ 3$. In this section we consider the problem: Given spaces $X, Y$ and $Z$, and a topology $\tau$ for $C(Y, Z)$, under what conditions does $(X, Y, Z, \tau)$ satisfy the partial exponential law? In an abstract sense this problem is answered for a very broad class of topologies by Theorem 1.1 of [33]; to obtain more concrete results we will restrict our attention to a "nice" class of topologies for $C(Y, Z)$, and seek results which will hold for every completely regular Hausdorff or every regular Hausdorff space $Z$. Notice that if $(X, Y, Z, \tau)$ satisfies the partial 
exponential law and if $\tau^{\prime} \leqq \tau$, then $\left(X, Y, Z, \tau^{\prime}\right)$ also satisfies the partial exponential law, so that restricting the topologies considered does not involve so great a loss of generality as might first appear.

For a cover, $\alpha$, of $Y$ let $\tau_{\alpha}$ denote the set-open topology generated by $\alpha$, i.e., the topology with subbase $\{N(A, U): A \in \alpha$ and $U \subseteq Z$ is open $\}$ where $N(A, U)$ $=\{f: f(A) \subseteq U\}$ and let $\tau_{\alpha}^{*}$ denote the topology with subbase $\{G(A, U): A \in \alpha$ and $U$ is an open subset of $A \times Z$ \} where $G(A, U)$ is the set of functions $f$ for which the graph of $\left.f\right|_{A}$ is contained in $U$. Define $\tau_{\alpha}^{\prime \prime}$ analogously to $\tau_{\alpha}^{*}$ using cozero sets instead of open sets. When $Z=R$ and $\{Y\} \in \alpha, \tau_{\alpha}^{\prime \prime}$ is the Moore topology on $C(Y)$ (see [33]). Also, where $Z$ is completely regular let $\tau_{\alpha}^{\prime}$ denote the topology of uniform convergence on members of $\alpha$ defined as in [25] with respect to any compatible uniformity of $Z$. (The topology $\tau_{\alpha}^{\prime}$ is independent of the particular compatible uniformity chosen.) To remain consistent with our previous notation, we let $p$ denote the cover of a space consisting of its points, and we let $k$ denote the cover consisting of the compact subsets. Recall that $\tau_{k}$, the compact-open topology, is equal to $\tau_{k}^{\prime}$ when the latter is defined, and note that $\tau_{k}=\tau_{k}^{*}$ when $Z$ is Hausdorff. (In general, $\tau_{\alpha} \leqq \tau_{\alpha}^{*}$ and $\tau_{\alpha}^{\prime} \leqq \tau_{\alpha}^{\prime \prime} \leqq \tau_{\alpha}^{*}$ but none of the remaining implications need hold-see [33].)

The following two results are easy consequences of [33, Theorem 1.1]. Recall that a function is closed if it maps closed sets to closed sets and that a subset of $X$ is a zero set if it is the inverse image of zero under some continuous real-valued function on $X$.

2.1 THEOREM. Let $X$ and $Y$ be arbitrary, let $\alpha$ be a cover of $Y$ and (for each $Z$ ) let $\tau$ be a topology for $C(Y, Z)$ satisfying $\tau_{\alpha} \leqq \tau \leqq \tau_{\alpha}^{*}$. Then the following three conditions are equivalent:

(i) For each space $Z,(X, Y, Z, \tau)$ satisfies the partial exponential law.

(ii) For $Z=X \times Y,(X, Y, Z, \tau)$ satisfies the partial exponential law.

(iii) For each $A \in \alpha$, the projection $\pi_{X}: X \times A \rightarrow X$ is closed.

2.2 THeOREM. Let $X$ and $Y$ be arbitrary, let $\alpha$ be a cover of $Y$, and (for each completely regular $Z$ ) let $\tau$ be a topology on $C(Y, Z)$ satisfying $\tau_{\alpha}^{\prime} \leqq \tau \leqq \tau_{\alpha}^{\prime \prime}$. Then the following three conditions are equivalent:

(i) For each completely regular Hausdorff space $Z,(X, Y, Z, \tau)$ satisfies the partial exponential law.

(ii) For some completely regular Hausdorff space $Z$ which contains a nondegenerate arc, $(X, Y, Z, \tau)$ satisfies the partial exponential law.

(iii) For each $A \in \alpha$ and each zero set $F$ of $X \times Y, \pi_{X}: X \times A \rightarrow X$ maps $F \cap X \times A$ to a closed set.

Closed projections and $z$-closed projections (those which map zero sets to closed sets) have been studied in [14], [21], and [33] and the papers referenced therein. The condition on projections which appears in Theorem 2.2 has not been previously studied, but most of the known results on $z$-closed projections carry over easily to 
this case-those which we will need will be given below as soon as we have made the appropriate definitions.

Where $Y^{\prime}$ is understood to be a subspace of some space $Y$, a subset of $Y^{\prime}$ is called a relative zero set if it is the intersection with $Y^{\prime}$ of some zero set of $Y$. Each relative zero set of $Y^{\prime}$ is of course a zero set, but the converse may (and often does) fail. For $X^{\prime} \subseteq X$ and $Y^{\prime} \subseteq Y$, we call $\pi_{X^{\prime}}: X^{\prime} \times Y^{\prime} \rightarrow X^{\prime}$ relatively $z$-closed (with respect to $X \times Y$ ) if it maps each relative zero set of $X^{\prime} \times Y^{\prime}$ to a closed set. Now let $\aleph$ denote an infinite cardinal and recall that a point of a space is called $\boldsymbol{\aleph}$-discrete if each $\boldsymbol{N}$-fold intersection of neighborhoods of that point is itself a neighborhood. We call a point $<\boldsymbol{N}$-discrete if it is $\boldsymbol{\aleph}^{\prime}$-discrete for each infinite cardinal $\boldsymbol{\aleph}^{\prime}$ less than $\boldsymbol{\aleph}$. Generalizing the notion of pseudo-א-compactness, we call a subspace $Y^{\prime}$ of $Y$ relatively pseudo- $\boldsymbol{K}$-compact (in $Y$ ) if each for each family $\left\{U_{\alpha}\right\}$ of disjoint nonempty cozero sets of $Y$ which has no cluster points, fewer than $\boldsymbol{W}$ of the $U_{\alpha}$ meet $Y^{\prime}$. (A cozero set is the complement of a zero set. We have used "cozero set" here in place of the to be expected "open set" in order to avoid having to assume that $Y$ be completely regular.) A subset $Y^{\prime}$ of $Y$ is called relatively pseudocompact if each continuous real-valued function on $Y$ maps $Y^{\prime}$ to a bounded set. From these definitions, the following proposition is immediate:

2.3 Proposition. (i) $A$ subset of $Y$ is relatively pseudo- $\boldsymbol{\aleph}_{0}$-compact if and only if it is relatively pseudocompact.

(ii) If $Y^{\prime} \subseteq Y$ is relatively pseudo- $\aleph$-compact, then so is each subset of the closure of $Y^{\prime}$.

(iii) If $Y$ is topologically complete, then each closed relatively pseudocompact subset of $Y$ is compact.

2.4 Theorem. Let $X^{\prime} \subseteq X, Y^{\prime} \subseteq Y$, and suppose $\pi_{X^{\prime}}: X^{\prime} \times Y^{\prime} \rightarrow X^{\prime}$ is relatively z-closed. Then:

(i) For each infinite cardinal $\boldsymbol{\aleph}$, either $Y^{\prime}$ is relatively pseudo- $\aleph$-compact or each point of $X^{\prime}$ is $\mathbf{N}$-discrete.

(ii) There exists an infinite cardinal $\aleph$ such that $Y^{\prime}$ is relatively pseudo-א-compact and each point of $X^{\prime}$ is $<\boldsymbol{N}$-discrete.

Proof. Part (i) follows from the obvious adaptation of the proof of [33, Theorem 3.1] and Part (ii) is an immediate consequence of Part (i).

2.5 THEOREM. Let $X^{\prime} \subseteq X$ and $Y^{\prime} \subseteq Y$ be relatively pseudo-N-compact, and suppose that each point of $X^{\prime}$ is $<\boldsymbol{\aleph}$-discrete. If $X^{\prime} \times Y^{\prime}$ is relatively pseudo-א-compact, then $\pi_{X^{\prime}}: X^{\prime} \times Y^{\prime} \rightarrow X^{\prime}$ is relatively z-closed. If $X$ is completely regular Hausdorff and some point of $X^{\prime}$ is not isolated, the converse holds.

Proof. The first assertion follows by the obvious generalization of the proofs of [21, Theorems 4.2 and 4.3] with the proof of 4.2 adapted so that the sets $Y_{k}$, and hence $Z_{k}$, will be zero sets of $X \times Y$. The second assertion follows by the obvious 
generalizations of the proof of [33, Lemma 3.3] and the first part of the proof of [33, Theorem 3.4].

The theorem above raises the question: Under what conditions is $X^{\prime} \times Y^{\prime}$ relatively pseudo- $\boldsymbol{\aleph}$-compact? Of the cases with $\boldsymbol{\aleph}>\boldsymbol{\aleph}_{0}$, which seem of limited interest, we only mention that [21, Corollary 4.6] generalizes at least for $X$ and $Y$ completely regular Hausdorff. For the case $\boldsymbol{N}=\boldsymbol{\aleph}_{0}$ we have a much stronger result, again an easy generalization of known results. Let $N$ denote the set of positive integers.

2.6 THEOREM. Let $X$ be completely regular Hausdorff and let $X^{\prime}$ be a subset of $X$. Then the following three conditions are equivalent:

(i) For each space $Y$ and each relatively pseudocompact subspace $Y^{\prime}$ of $Y, X^{\prime} \times Y^{\prime}$ is relatively pseudocompact in $X \times Y$.

(ii) For each completely regular Hausdorff pseudocompact space $Y, X^{\prime} \times Y$ is relatively pseudocompact in $X \times Y$.

(iii) If $U$ is any family of disjoint nonempty open subsets of $X$ each of which meet $Y^{\prime}$, then there exists an infinite subfamily $\left\{U_{n}: n \in N\right\}$ such that for each filter $\phi$ consisting of infinite subsets of $N, \bigcap_{F \in \phi} \operatorname{cl}_{X}\left(\bigcup_{n \in F} U_{n}\right) \neq \varnothing$.

Proof. The implication (i) $\Rightarrow$ (ii) is trivial and the implications (ii) $\Rightarrow$ (iii) and (iii) $\Rightarrow$ (i) follow by the obvious adaptation of the proof of [17, Theorem 3.6].

Note that with $X=X^{\prime}$, each compact Hausdorff space satisfies the conditions in the theorem above. (For a discussion of other spaces with this property, see [34]. Many of these results generalize to the case $X^{\prime} \neq X$.) From Theorems 2.2, 2.4, 2.5 and 2.6 we have:

2.7 ThEOREM. Let $\alpha$ and $\tau$ be as in Theorem 2.2. Then $(X, Y, Z, \tau)$ satisfies the partial exponential law for each compact Hausdorff space $X$ and each completely regular Hausdorff space $Z$ (or some completely regular Hausdorff space $Z$ which contains a nondegenerate arc) if and only if each $A$ in $\alpha$ is relatively pseudocompact.

2.8 THEOREM. Let $\alpha$ and $\tau$ be as in Theorem 2.1, and let $Y$ be regular Hausdorff. Then $(X, Y, Z, \tau)$ satisfies the partial exponential law for each space $X$ (or each compact Hausdorff space $X$ ) and each regular Hausdorff space $Z$ (or for $Z=Y$ ) if and only if each $A$ in $\alpha$ is compact.

Proof. This follows by 2.1 and the fact (see [33]) that $\pi_{X}: X \times A \rightarrow X$ is closed for each space $X$ (or each compact Hausdorff space $X$ ) if and only if $A$ is compact.

3. The exponential law. In this section we treat the remaining inclusion of the exponential law, $C(X \times Y, Z) \supseteq \mu^{-1}\left(C\left(X, C(Y, Z)_{\tau}\right)\right)$, and the exponential law itself. Note that if this inclusion holds for a topology $\tau$, then it also holds for each topology greater than $\tau$. Again we restrict attention to the topologies $\tau_{\alpha}$ and $\tau_{\alpha}^{\prime}$, and we will also restrict the covers, $\alpha$, considered. For $\alpha$ a cover of $Y$ and $Z$ a topological space we call $\alpha Z$-full if for each $A \in \alpha, \alpha$ contains a base for the weak 
topology on $A$ induced by $C(Y, Z)$. Note that if $\alpha$ is a cover and $\alpha^{\prime}=\left\{A^{\prime}: A^{\prime} \subseteq A\right.$ for some $A \in \alpha\}$, then $\tau_{\alpha}^{\prime}=\tau_{\alpha^{\prime}}^{\prime}$. Thus when dealing with the topology $\tau_{\alpha}^{\prime}$ we may suppose that $\alpha$ is $Z$-full. For a cover of $Y$ let $S_{\alpha}(X \times Y, Z)$ denote the set of functions in $F(X \times Y, Z)$ whose restrictions to sets of the form $\{x\} \times Y \cup X \times A$ are continuous for each $x$ in $X$ and each $A$ in $\alpha$.

3.1 Theorem. Let $X, Y$, and $Z$ be arbitrary and let $\alpha$ be a cover of $Y$. Then a function $f$ in $F(X \times Y, Z)$ is in $\mu^{-1}\left(C\left(X, C(Y, Z) \tau_{\alpha}\right)\right)$ if and only if $f$ satisfies the following two conditions:

(i) For each $x$ in $X,\left.f\right|_{\{x\} \times Y}$ is continuous.

(ii) For each $A \in \alpha$ and each closed $H \subseteq Z, \pi_{X}\left(f^{-1}(H) \cap X \times A\right)$ is closed.

Furthermore, if $\alpha$ is Z-full, each such $f$ is in $S_{\alpha}(X \times Y, Z)$.

Proof. The condition (i) is clearly equivalent to the condition $\mu f \in F(X, C(Y, Z))$. Since $(\mu f)^{-1}(N(A, U))=X \mid \pi_{X}\left(f^{-1}(Z \backslash U) \cap X \times A\right)$, the second condition is equivalent to the continuity of $\mu f: X \rightarrow C(Y, Z)_{\tau_{\alpha}}$. Finally, to see that $\left.f\right|_{X \times A}$ is continuous when $\mu f$ is continuous, $A$ is in $\alpha$ and $\alpha$ is $Z$-full, note that if $A^{\prime}$ is a neighborhood in $A$ of $y$ and if $A^{\prime}$ is contained in $\mu f(x)^{-1}(U)$, then $(\mu f)^{-1}\left(N\left(A^{\prime}, U\right)\right) \times A^{\prime}$ is contained in $f^{-1}(U)$ and is a neighborhood of $(x, y)$ in $X \times A$.

For $Z$ completely regular, $A \subseteq Y$, and $f \in C(Y, Z)$, let

$$
U(f, A)=\{\{(y, z) \in A \times Z: d(z, f(y))<\varepsilon)\}: d: Z \times Z \rightarrow R
$$

is a continuous pseudometric and $\varepsilon>0\}$

and for $U$ in $U(f, A)$ let $N^{\prime}(U)=\left\{g \in C(Y, Z)\right.$ : the graph of $\left.g\right|_{A}$ is contained in $\left.U\right\}$. Then the collection $\left\{N^{\prime}(U): U \in U(f, A), A \in \alpha\right\}$ form a (not in general open) neighborhood base for $f$ in $C(Y, Z)_{\tau_{\alpha}^{\prime}}$ (see [33]). For $f: X \times Y \rightarrow Z$, let $\Gamma_{f}$ be the map $\Gamma_{f}(x, y)=(y, f(x, y))$ and note that $\Gamma_{f}$ is continuous wherever $f$ is continuous.

3.2 Theorem. Let $X$ and $Y$ be arbitrary, let $Z$ be completely regular, and let $\alpha$ be a cover of $Y$. Then a function $f$ in $F(X \times Y, Z)$ is in $\mu^{-1}\left(C\left(X, C(Y, Z)_{\tau_{\alpha}}\right)\right)$ if and only if $f$ satisfies the following two conditions:

(i) For each $x$ in $X,\left.f\right|_{\{x\} \times Y}$ is continuous.

(ii) For each $A$ in $\alpha$, each $x_{0}$ in $X$ and each $U$ in $U\left(\mu f\left(x_{0}\right), A\right), X \mid \pi_{X}\left(X \times A \backslash \Gamma_{f}^{-1}(U)\right)$ is a neighborhood of $x_{0}$. Thus $\mu^{-1}\left(C\left(X, C(Y, Z)_{\tau_{\alpha}}^{\prime}\right)\right)$ is contained in $S_{\alpha}(X \times Y, Z)$.

Proof. In view of the preceding comments, the first statement follows by the obvious modification of the proof of Theorem 3.1. To see that $\mu^{-1}\left(C\left(X, C(Y, Z)_{\tau_{\alpha}^{\prime}}\right)\right)$ $\subseteq S_{\alpha}(X \times Y, Z)$, let $f \in \mu^{-1}\left(C\left(X, C(Y, Z)_{\tau_{\alpha}}^{\prime}\right)\right)$, let $A \in \alpha$, let $\left(x_{0}, y_{0}\right) \in X \times A$, and let $U$ be a neighborhood of $f\left(x_{0}, y_{0}\right)$, say $U=\left\{z: d\left(z, f\left(x_{0}, y_{0}\right)\right)<\varepsilon\right\}$ for $d$ a continuous pseudometric and $\varepsilon>0$. Choose a neighborhood $A^{\prime}$ of $y_{0}$ in $A$ such that for $y \in A^{\prime}$, $d\left(f\left(x_{0}, y\right), f\left(x_{0}, y_{0}\right)\right)<\varepsilon / 2$ set $U^{\prime}=\left\{(y, z) \in A \times Z: d\left(f\left(x_{0}, y\right), z\right)<\varepsilon / 2\right\}$, and note that for $V=X \mid \pi_{X}\left(X \times A \mid \Gamma_{f}^{-1}\left(U^{\prime}\right)\right), V \times A^{\prime}$ is a neighborhood of $\left(x_{0}, y_{0}\right)$ (in $\left.X \times A\right)$, which is contained in $f^{-1}(U)$. 
By the above two theorems, a sufficient condition, where $\tau=\tau_{\alpha}^{\prime}$ or $\tau=\tau_{\alpha}$ and $\alpha$ is $Z$-full, that $C(X \times Y, Z) \supseteq \mu^{-1}\left(C\left(X, C(Y, Z)_{\tau}\right)\right)$ is that $S_{\alpha}(X \times Y, Z)=C(X \times Y, Z)$. In many cases this condition is also necessary; for instance, if $\tau=\tau_{\alpha}$ and $\pi_{X}: X \times A$ $\rightarrow X$ is closed for each $A$ in $\alpha$, or if $\tau=\tau_{\alpha}^{\prime}$ and $\pi_{X}: X \times A \rightarrow X$ is $z$-closed for each $A$ in $\alpha$, then $S_{\alpha}(X \times Y, Z)=\mu^{-1}\left(C\left(X, C(Y, Z)_{\tau}\right)\right)$.

The proofs of Theorems 3.1 and 3.2 generalize easily to yield our next result. For $\alpha$ a cover of $Y$ let $C_{\alpha}(Y, Z)$ denote the set of functions in $F(Y, Z)$ whose restriction to each member of $\alpha$ is continuous. For covers $\alpha$ and $\beta$, let $\alpha \times \beta$ denote $\{A \times B: A \in \alpha, B \in \beta\}$.

3.3 Theorem. Let $X, Y$, and $Z$ be arbitrary, let $\alpha$ and $\alpha^{\prime}$ be covers of $Y$ and let $\alpha^{\prime \prime}$ be a cover of $X$. Set $\beta=p \times \alpha^{\prime}$ and $\beta^{\prime}=\beta \cup\left(\alpha^{\prime \prime} \times \alpha\right)$. Then

(i) $\mu^{-1}\left(C_{\alpha^{\prime \prime}}\left(X, C_{\alpha^{\prime}}(Y, Z)_{\tau_{\alpha}}\right)\right)=\left\{f \in C_{\beta}(X \times Y, Z)\right.$ : For each $A^{\prime \prime} \times A \in \alpha^{\prime \prime} \times \alpha$ and each closed $H \subseteq Z, \pi_{A^{\prime \prime}}\left(f^{-1}(H) \cap A^{\prime \prime} \times A\right)$ is closed $\}$.

(ii) If $\alpha$ is $Z$-full, $\mu^{-1}\left(C_{\alpha^{\prime \prime}}\left(X, C_{\alpha^{\prime}}(Y, Z)_{\tau_{\alpha}}\right)\right) \subseteq C_{\beta^{\prime}}(X \times Y, Z)$.

(iii) If for each $A^{\prime \prime} \times A$ in $\alpha^{\prime \prime} \times \alpha, \pi_{A^{\prime \prime}}: A^{\prime \prime} \times A \rightarrow A^{\prime \prime}$ is closed, then

$$
\mu^{-1}\left(C_{\alpha^{\prime \prime}}\left(X, C_{\alpha^{\prime}}(Y, Z)_{\tau_{\alpha}}\right)\right) \supseteq C_{\beta^{\prime}}(X \times Y, Z) .
$$

Also, for $Z$ completely regular,

(i') $\mu^{-1}\left(C_{\alpha^{\prime \prime}}\left(X, C_{\alpha^{\prime}}(Y, Z)_{\tau_{\alpha}^{\prime}}\right)\right)=\left\{f \in C_{\beta}(X \times Y, Z)\right.$ : For each $A^{\prime \prime} \times A \in \alpha^{\prime \prime} \times \alpha$, each $x_{0}$ in $A^{\prime \prime}$ and each $U$ in $U\left(\mu f\left(x_{0}\right), A\right), A^{\prime \prime} \mid \pi_{A^{\prime \prime}}\left(A^{\prime \prime} \times A \backslash \Gamma_{f}^{-1}(U)\right)$ is a neighborhood of $\left.x_{0}\right\}$.

(ii') $\mu^{-1}\left(C_{\alpha^{\prime \prime}}\left(X, C_{\alpha^{\prime}}(Y, Z)_{\tau_{\alpha}^{\prime}}\right)\right) \subseteq C_{\beta^{\prime}}(X \times Y, Z)$.

(iii) If for each $A^{\prime \prime} \times A$ in $\alpha^{\prime \prime} \times \alpha, \pi_{A^{\prime \prime}}: A^{\prime \prime} \times A \rightarrow A^{\prime \prime}$ is $z$-closed, then

$$
\mu^{-1}\left(C_{\alpha^{\prime \prime}}\left(X, C_{\alpha^{\prime}}(Y, Z)_{\tau^{\prime}}\right)\right) \supseteq C_{\beta^{\prime}}(X \times Y, Z) .
$$

(iv) If $C_{\alpha^{\prime \prime}}\left(X, C_{\alpha^{\prime}}(Y, Z)\right)=C\left(X, C(Y, Z)_{\tau^{\prime}}\right)$ and if for each $A^{\prime \prime} \times A$ in $\alpha^{\prime} \times \alpha$, $\pi_{A^{\prime \prime}}: A^{\prime \prime} \times A \rightarrow A^{\prime \prime}$ is relatively $z$-closed (in $X \times Y$ ), then

$$
\mu^{-1}\left(C_{\alpha^{\prime \prime}}\left(X, C_{\alpha^{\prime}}(Y, Z)_{\tau_{\alpha}^{\prime}}\right)\right) \supseteq C_{\beta^{\prime}}(X \times Y, Z) .
$$

We will now use Theorem 3.3 to give conditions under which the relation $S_{\alpha}(X \times Y, Z)=C(X \times Y, Z)$ holds. Where $\mathscr{S}$ is a family of spaces, and $\alpha$ is a cover of $Y$, we say that $\alpha \mathscr{S}$-generates $Y$ if $C_{\alpha}(Y, Z)=C(Y, Z)$ for each $Z$ in $\mathscr{S}$. If $\alpha$ $\mathscr{S}$-generates $Y$ for $\mathscr{S}$ the class of all spaces, $\alpha$ is said to generate $Y$. A cover of a space which contains a neighborhood base for each of its points will be called a local cover. Note that if $\{Y\}$ is in $\alpha$, if $\alpha$ is a local cover of $Y$, or if $\alpha$ is a closed locally finite cover of $Y$, then $S_{\alpha}(X \times Y, Z)=C(X \times Y, Z)$.

3.4 THEOREM. Let $\mathscr{S}$ be a family of spaces, let $\alpha$ be a cover of $Y$ which $\mathscr{S}$-generates $Y$ and let $\alpha^{\prime}$ be a cover of $X$ such that $\{X\}$ is in $\alpha^{\prime}$ or $\alpha^{\prime}$ is a local cover or $\alpha^{\prime}$ is a closed locally finite cover. If either of the following two conditions are satisfied, then for each $Z$ in $\mathscr{S}, S_{\alpha}(X \times Y, Z)=C_{\alpha^{\prime} \times \alpha}(X \times Y, Z)=C(X \times Y, Z)$ : 
(i) $C(X, Z)_{\tau_{\alpha^{\prime}}}$ is in $\mathscr{S}$ whenever $Z$ is in $\mathscr{S}$, and for each $A \times A^{\prime}$ in $\alpha \times \alpha^{\prime}$ the projection $\pi_{A}: A \times A^{\prime} \rightarrow A$ is closed.

(ii) Each space in $\mathscr{S}$ is completely regular, $C(X, Z)_{\tau_{\alpha^{\prime}}}$ is in $\mathscr{S}$ whenever $Z$ is in $\mathscr{S}$, and for each $A \times A^{\prime}$ in $\alpha \times \alpha^{\prime}$ the projection $\pi_{A}: A \times A^{\prime} \rightarrow A$ is relatively z-closed.

Proof. By Theorem 3.3 and observation, we have, for $Z$ in $\mathscr{S}$ :

$$
\begin{aligned}
C(Y \times X, Z) & \subseteq C_{\alpha \times \alpha^{\prime}}(Y \times X, Z)=\mu^{-1}\left(C_{\alpha}\left(Y, C(X, Z)_{\tau}\right)\right)=\mu^{-1}\left(C\left(Y, C(X, Z)_{\tau}\right)\right) \\
& \subseteq S_{\alpha^{\prime}}(Y \times X, Z)=C(Y \times X, Z),
\end{aligned}
$$

where $\tau$ is $\tau_{\alpha}$ or $\tau_{\alpha}^{\prime}$ according as (i) or (ii) is satisfied. Thus

$$
C(Y \times X, Z)=C_{\alpha \times \alpha^{\prime}}(Y \times X, Z),
$$

i.e., $C(X \times Y, Z)=C_{\alpha^{\prime} \times \alpha}(X \times Y, Z)$. Finally, since

$$
C(X \times Y, Z) \subseteq S_{\alpha}(X \times Y, Z) \subseteq C_{\alpha^{\prime} \times \alpha}(X \times Y, Z), \quad S_{\alpha}(X \times Y, Z)=C(X \times Y, Z) .
$$

If $p \subseteq \alpha$ and $\mathscr{S}$ is the class of all $T_{0}, T_{1}$, or Hausdorff spaces, or if $p \subseteq \alpha \subseteq k$ and $\mathscr{S}$ is the class of all regular Hausdorff spaces, then the conditions on $\mathscr{S}$ in part (i) of the theorem above will be satisfied. For simplicity we will only give corollaries for the two cases: $\mathscr{S}=\mathscr{S}_{0}$, the class of all spaces and $\mathscr{S}=\mathscr{S}_{1}$, the class of all completely regular spaces, but, of course corresponding results hold for the classes mentioned above.

Let $s$ denote the cover of a space consisting of all its convergent sequences (together with their limit points) and recall that a space is called a $k$-space (resp., a sequential space) if it is generated by $k$ (resp., $s$ ). A space which is $\mathscr{S}_{1}$-generated by $k$ is called a $k_{R}$-space. For $P$ a topological property, we take " $X$ is locally $P$ " to mean that the family of subspaces of $X$ which have $P$ form a local cover. Recall that a space is called $\boldsymbol{X}$-compact if each open cover admits a subcover of cardinality less than $\boldsymbol{\aleph}$. For the definition of weakly $\boldsymbol{\aleph}$-compact, see [21].

3.5 Corollary. If $Y$ is $\mathscr{S}$-generated by $\alpha$ and $X, Y, \alpha$ and $\mathscr{S}$ satisfy any one of the following conditions, then for each $Z$ in $\mathscr{S}, S_{\alpha}(X \times Y, Z)=C(X \times Y, Z)$.

(i) $\mathscr{S}=\mathscr{S}_{0}$ or $\mathscr{S}_{1}$ and $X$ is locally compact.

(ii) $\mathscr{S}=\mathscr{S}_{0}$ or $\mathscr{S}_{1}, X$ is locally countably compact and each member of $\alpha$ can be embedded in a sequential space.

(iii) $\mathscr{S}=\mathscr{S}_{1}, X$ is pseudocompact or locally relatively pseudocompact, each member of $\alpha$ is closed and relatively pseudocompact, and $Y$ is a $k$-space.

(iv) $\mathscr{S}=\mathscr{S}_{1}, X$ is pseudocompact or locally relatively pseudocompact and $\alpha \subseteq k$.

(v) $\mathscr{S}=\mathscr{S}_{0}$ or $\mathscr{S}_{1}, X$ is locally $\boldsymbol{\aleph}$-compact and each member of $\alpha$ is $<\boldsymbol{\aleph}$-discrete.

(vi) $\mathscr{S}=\mathscr{S}_{1}, X$ is weakly $\aleph$-compact or locally weakly $\aleph$-compact and each member of $\alpha$ is $<\boldsymbol{\aleph}$-discrete.

Proof. All that is needed is to show that the appropriate projections are, for (i), (ii) and (v) closed and for (iii), (iv) and (vi), $z$-closed. For (i) and (v) this is well known (see for instance [33]), for (ii) this is established in [14], and for (iii) and (iv) 
this follows from the fact that the product of a pseudocompact space with a pseudocompact $k$-space is pseudocompact (see for instance [32]) and the fact that each closed subspace of a $k$-space is a $k$-space. For (vi), this is established in [21].

The strength of Corollary 3.5 is perhaps best indicated by the fact that for $\mathscr{S}=\mathscr{S}_{0}$, the converses of (i) with $\alpha=k$ and of (ii) with $\alpha=s$ both hold. This is (essentially) proved in [27]. For another corollary of Theorem 3.4, and a converse to (iv) with $\alpha=k$, see [35].

4. Ascoli theorems. In this section we will apply the results of the previous sections to prove several Ascoli and weak Ascoli theorems in the sense that we have been using these terms. However, our results actually encompass a broader class of theorems: Let w. $A(P)$ (respectively, $A(P)$ ) denote the statement obtained by replacing even continuity in the statement of the weak Ascoli theorem (respectively, Ascoli theorem) by a property $P$. If $(Y, Z, \tau)$ satisfies the weak Ascoli theorem and $P$ satisfies the condition: Each $\tau$-closed, pointwise bounded subset of $C(Y, Z)$ which has $P$ is evenly continuous, then $(Y, Z, \tau)$ satisfies w. $A(P)$. If, in addition, $(Y, Z, \tau)$ satisfies the Ascoli theorem and $P$ satisfies the further requirement: Each $\tau$-compact evenly continuous subset of $C(Y, Z)$ has $P$, then $(Y, Z, \tau)$ satisfies $A(P)$. For $Z$ regular Hausdorff and $\tau \geqq \tau_{p}$, the following properties satisfy both of the above conditions on $P$ (when they are defined; the equicontinuity conditions are only defined when $Z$ is a uniform space): The property used in the "Ascoli theorem" of [18]; the "property $\alpha$ " of [45], equicontinuity (in the classical sense), $B$-equicontinuity (of [45]), and equicontinuity in the sense of [6]. (For proofs of the above nontrivial assertions, see [45], an excellent study of the relationships among these properties.)

In view of the comments above, the results which we are about to give will generalize all of the "Ascoli theorems" of the form w. $A(P)$ and $A(P)$ which use these properties and are known to the author. Let $\beta$ denote the cover consisting of all relatively pseudocompact subsets.

4.1 TheOREM. Let $Y$ be arbitrary, and let $\tau$ denote a topology on $C(Y, Z)$.

(i) If $\tau \leqq \tau_{k}$ and $Z$ is regular Hausdorff, then $(Y, Z, \tau)$ satisfies the weak Ascoli theorem.

(ii) If $Y$ is regular Hausdorff and $\alpha$ is a cover of $Y$ such that for each regular Hausdorff space $Z,\left(Y, Z, \tau_{\alpha}\right)$ satisfies the weak Ascoli theorem, then $\alpha \subseteq k$.

(iii) If $\tau \leqq \tau_{\beta}^{\prime \prime}$ and $Z$ is completely regular Hausdorff, then $(Y, Z, \tau)$ satisfies the weak Ascoli theorem.

(iv) If $\alpha$ is a cover of $Y$ such that, for some completely regular Hausdorff space $Z$ which contains a nondegenerate arc, either $\left(Y, Z, \tau_{\alpha^{\prime}}\right)$ or $\left(Y, Z, \tau_{\alpha}\right)$, satisfies the weak Ascoli theorem, then $\alpha \subseteq \beta$.

Proof. These results follow from 1.2, 2.7, and 2.8.

The requirement in part (iv) that $Z$ contain a nondegenerate arc cannot be weakened. If $Z$ contains no nondegenerate arc and $Y$ is arc-wise connected, then 
$C(Y, Z)$ is just the set of constant functions from $Y$ to $Z$ so for any pair $\alpha, \alpha^{\prime}$ of covers of $Y, \tau_{\alpha}^{\prime}=\tau_{\alpha}^{\prime}$.

Note that given $Y$ and $Z$ there is a unique largest topology, $\tau_{0}$, for which $\left(Y, Z, \tau_{0}\right)$ satisfies the weak Ascoli theorem. (The topology $\tau_{0}$ is characterized by the property: $F \subseteq C(Y, Z)$ is $\tau_{0}$-closed if and only if $F \cap K$ is relatively closed in $K$ for each $\tau_{p}$-compact evenly continuous subset $K$ of $C(Y, Z)$.) If $(Y, Z, \tau)$ satisfies the Ascoli theorem (for $\tau \geqq \tau_{p}$ ) then $\tau_{0}$ is the $k$-extension of $\tau$ (for definitions of the terms $k$-extension and $k$-space, see [25]) so if $C(Y, Z)_{\tau}$ is a $k$-space, $\tau=\tau_{0}$. In particular, if $Y$ is a hemicompact $k$-space and $Z$ is metrizable, (so $C(Y, Z)_{\tau_{k}}$ is metrizablesee [1]) then $\tau_{0}=\tau_{k}$. Note that parts (ii) and (iv) of Theorem 4.1 provide partial converses to the results below.

4.2 THEOREM. Let $Y$ be arbitrary and let $\alpha$ be a cover of $Y$.

(i) If $\alpha \subseteq k$, if $C_{\alpha}(Y, Z)=C(Y, Z)$ for each regular Hausdorff space $Z$, and if $Z^{\prime}$ is a regular Hausdorff space for which $\alpha$ is $Z^{\prime}$-full, then for any topology $\tau$ satisfying $\tau_{\alpha} \leqq \tau \leqq \tau_{k},\left(Y, Z^{\prime}, \tau\right)$ satisfies the Ascoli theorem.

(ii) If $\alpha \subseteq \beta$, if $C_{\alpha}(Y)=C(Y)$ and if $Z^{\prime}$ is a completely regular Hausdorff space for which $\alpha$ is $Z^{\prime}$-full, then for any topology satisfying $\tau_{\alpha} \leqq \tau \leqq \tau_{\beta}^{*},\left(Y, Z^{\prime}, \tau\right)$ satisfies the Ascoli theorem.

(iii) If $\alpha \subseteq \beta$, if $C_{\alpha}(Y)=C(Y)$ and if $Z$ is completely regular Hausdorff, then for any topology satisfying $\tau \leqq \tau_{\alpha}^{\prime} \leqq \tau_{\beta}^{\prime \prime},(Y, Z, \tau)$ satisfies the Ascoli theorem.

Proof. 1.3, 3.1, 3.2, 3.5 and 4.1.

4.3 COROLLARY. Let $Z$ be completely regular and let $\tau$ be the topology of uniform convergence.

(i) If $Y$ is pseudocompact, then $(Y, Z, \tau)$ satisfies the Ascoli theorem.

(ii) If $(Y, Z, \tau)$ satisfies the (weak) Ascoli theorem and $Z$ contains a nondegenerate arc, then $Y$ is pseudocompact.

Proof. For $\alpha=\{Y\}, \tau=\tau_{\alpha}^{\prime}$.

This result generalizes the Ascoli theorem of [19]. For our next corollary, recall that with "pointwise countable type" defined as in [32], each locally compact space, each first countable space and each space complete in the sense of Čech is of pointwise countable type-also, each space of pointwise countable type is a $k$-space. It should be pointed out that the space $Y$ in the following two corollaries need not be (in fact, will seldom be) a $k$-space.

4.4 COROLlaRY. If $Z$ is regular Hausdorff, and $Y$ is a product of spaces of pointwise countable type, then $\left(Y, Z, \tau_{k}\right)$ satisfies the Ascoli theorem.

Proof. It is shown in [33] (and [35]) that for such spaces $Y, C_{k}(Y, Z)=C(Y, Z)$ for each regular space $Z$. 
4.5 COROLlary. If $Z$ is completely regular Hausdorff and $Y=\Pi_{\lambda} X_{\lambda}$ where each $X_{\lambda}$ is locally relatively pseudocompact and satisfies $C_{k}\left(X_{\lambda}\right)=C\left(X_{\lambda}\right)$, and if $\tau_{k} \leqq \tau \leqq \tau_{\beta}^{\prime \prime}$, then $(Y, Z, \tau)$ satisfies the Ascoli theorem.

Proof. It is shown in [35] that for such spaces $Y, C_{k}(Y)=C(Y)$.

Corollaries 4.4 and 4.5 are new-in fact, the best previous result for $\tau_{k}$ is that $\left(Y, Z, \tau_{k}\right)$ satisfies the Ascoli theorem if $Y$ is a $k$-space and $Z$ is regular Hausdorff, proved in [5] and [37]. Of course, $Y$ is a $k$-space if and only if $C_{k}(Y, Z)=C(Y, Z)$ for each space $Z$.

We close this section with an example of a pair $(Y, Z)$ such that $(Y, Z, \tau)$ does not satisfy the Ascoli theorem for any topology $\tau$. In [41, Lemma 5.5] Spanier gives an example of spaces $Y$ and $Z$ such that for no topology $\tau$ does $(K, Y, Z, \tau)$ satisfy the exponential law for each compact (Hausdorff) space $K$. Unfortunately, neither of the spaces in this example is regular. Our example owes a debt both to Spanier's example and to [5].

4.6 EXAMPLE. There exists a completely regular Hausdorff space $Y$ such that if $Z$ is any space containing a nondegenerate arc, and $\tau$ is any topology on $C(Y, Z)$, $(Y, Z, \tau)$ does not satisfy the Ascoli theorem.

Proof. Without loss of generality we may suppose that $Z=I$, the unit interval. Let $\alpha$ be the collection of all closed subsets of sets of the form $\{0\} \cup\left\{S_{n}\right\} \cup[1 / m, 1]$ for $\left\{S_{n}\right\}$ a positive sequence converging to 0 and $m$ a positive integer. Then $\alpha$ is a cover of $I$ and $\alpha \subseteq k$, so on $C(I, I), \tau_{\alpha}=\tau_{\alpha}^{\prime} \leqq \tau_{k}$. In fact, $\tau_{\alpha}$ is strictly less than $\tau_{k}$, for if $A_{1}, \ldots, A_{n} \in \alpha$ and $U_{1}, \ldots, U_{n}$ are open in $I, \bigcup_{i=1}^{n} A_{i}$ is compact and has nonempty complement in $I$, so there exists a continuous function in $\bigcap_{i=1}^{n} N\left(A_{i}, U_{i}\right)$ which maps some point of $I$ to 1 . Thus the $\tau_{k}$-open set $N(I,[0,1))$ is not $\tau_{\alpha}$-open. It follows that $e: C(I, I)_{\tau_{\alpha}} \times I \rightarrow I$ is not continuous (otherwise the identity $\mu e: C(I, I)_{\tau_{\alpha}} \rightarrow C(I, I)_{\tau_{k}}$ would be continuous) but by [25, Theorem 7.10(e)] $e: C(I, I)_{\tau_{\alpha}} \times A \rightarrow I$ is continuous for each $A$ in $\alpha$.

Set $Y=C(I, I)_{\tau_{\alpha}}$. Since $\tau_{\alpha} \geqq \tau_{p}, Y$ is Hausdorff and since $Y$ is uniformizable, it is completely regular. Suppose there exists a topology $\tau$ such that $(Y, I, \tau)$ satisfies the Ascoli theorem. Then since $e^{\prime}: I \times Y \rightarrow I$ is not continuous, where $e^{\prime}(x, f)=f(x)$, $\mu e^{\prime}: I \rightarrow C(Y, I) \tau$ is not continuous. But since for each $A$ in $\alpha, e^{\prime}: A \times Y \rightarrow I$ is continuous, and since each such $A$ is compact, the restriction of $\mu e^{\prime}$ to each member of $\alpha$ is continuous. But obviously a function continuous on each member of $\alpha$ is continuous, so $\mu e^{\prime}$ is continuous and we have the desired contradiction.

Results such as the one above, and the one in [2] which shows that if $Y$ is not locally compact and $Z$ contains a nondegenerate arc, then there is no topology $\tau$ on $C(Y, Z)$ such that $(X, Y, Z, \tau)$ will satisfy the exponential law for each space $X$, can be (and are) interpreted as showing that the notion of a topological space is not sufficiently general to provide a basis for the investigation and use of the exponential map. Perhaps a more convincing example is [16] where, in order to carry out differential calculus in vector spaces without norm, the authors are forced 
to abandon topological spaces in order to assure that the exponential map will be well behaved. What appears to be the most satisfactory replacement, in this context, for topological spaces are convergence spaces (see [31] for references). Many of the results in this paper carry over directly to convergence spaces; see [39] for proofs of some of this.

5. Generalizations. In this section we consider various ways in which the technique introduced in $\$ 2$ can be generalized to produce theorems of Ascoli type other than the weak Ascoli and Ascoli theorems. Let us first note that, since the $\tau_{p}$-closure of an evenly continuous family is evenly continuous, all of our results can be restated so as to characterize the relatively compact subsets of $C(Y, Z)$ (those whose closures are compact). Next, note that if $(Y, Z, \tau)$ satisfies the weak Ascoli theorem or the Ascoli theorem, then so (in the obvious sense) does any closed (or $k$-closed-meets each compact subset in a closed set) subspace of $C(Y, Z)_{\tau}$. Thus our results yield Ascoli type theorems for spaces of uniformly continuous functions, spaces of homomorphisms, etc., but they do not yield all such theorems. However, for the space of continuous functions which preserve base points, and with the Cartesian product replaced by the smashed product, Theorems 1.2 and 1.3 carry over directly.

In a different vein, Theorems 1.2 and 1.3 carry over if even continuity is replaced by even continuity on a cover, $\alpha$ (in the sense of [25]) and $C(Y, Z)$ is replaced by $C_{\alpha}(Y, Z)$. In particular, for $\alpha=k$ (which is probably the only case of interest) this yields the following result:

5.1 THEOREM. Let $Z$ be regular Hausdorff, $Y$ arbitrary. Then the following two conditions are equivalent:

(i) A subset of $C(Y, Z)$ is $\tau_{k}$-compact if and only if it is $\tau_{k}$-closed, pointwise bounded and evenly continuous on compacta.

(ii) $C(Y, Z)$ is $k$-closed in $C_{k}(Y, Z)_{\tau_{k}}$.

Proof. By the remarks above and Theorem 3.3, a subset of $C_{k}(Y, Z)$ is $\tau_{k^{-}}$compact if and only if it is $\tau_{k}$-closed, pointwise bounded and evenly continuous on compacta. Thus (ii) $\Rightarrow$ (i) is clear and (i) $\Rightarrow$ (ii) follows from the observation that if $C(Y, Z)$ is not $k$-closed in $C_{k}(Y, Z)_{\tau_{k}}$, then there exists a compact $K \subseteq C_{k}(Y, Z)_{\tau_{k}}$ such that $K \cap C(Y, Z)$, which is $\tau_{k}$-closed in $C(Y, Z)$, pointwise bounded and evenly continuous on compacta, is not compact.

Note that if $Y$ is a $k$-space, or, more generally, if $C_{k}(Y, Z)=C(Y, Z)$, then $Y$ satisfies (ii) and hence (i) of the theorem above. In particular, product spaces like those in Corollaries 4.4 and 4.5 will satisfy (i) for suitable spaces $Z$.

Before considering other conditions which can be used to replace even continuity, let us examine the role played by even continuity, and the separation axioms in the proofs of Theorems 1.2 and 1.3. Recall that a subspace $F$ of $C(Y, Z)_{\tau}$ is called jointly continuous if $e: F \times Y \rightarrow Z$ is continuous. If $F$ is jointly continuous 
as a subspace of $C(Y, Z)_{\tau_{p}}$, we call $F e$-continuous. In the first part of the proof of 1.2 , even continuity wherever it appears is equivalent to $e$-continuity, and the only use of the separation axioms is in the assertion that $\tau_{p}$ and $\tau$ coincide on $\mu f(k)$ since both are compact, which requires the Hausdorff axiom. In the second portion of this proof, even continuity and regularity are used to guarantee that the $\tau_{p^{-}}$ closure in $F(Y, Z)$ of a pointwise bounded, $e$-continuous subset is $e$-continuous, but not elsewhere, and the Hausdorff axiom is not used. In the proof given below Theorem 1.3, the Hausdorff axiom is used in both parts but no essential use is made either of even continuity (as opposed to $e$-continuity) or of regularity. We summarize these observations below.

5.2 THEOREM. Let $\tau \geqq \tau_{p}$. Then the conditions below are related as follows: (i) $\Rightarrow$ (ii); (ii) $\Rightarrow$ (i) if $K$ and $Z$ are assumed to be Hausdorff, and (iii) $\Leftrightarrow$ (iv) if $Z$ is Hausdorff.

(i) If $K$ is compact, $(K, Y, Z, \tau)$ satisfies the partial exponential law.

(ii) If $K \subseteq C(Y, Z)$ is $\tau$-closed and pointwise bounded, and if the $\tau_{p}$-closure in $F(Y, Z)$ of $K$ is e-continuous, then $K$ is $\tau$-compact.

(iii) If $K$ is compact Hausdorff, then $C(K \times Y, Z) \supseteq \mu^{-1}\left(C\left(K, C(Y, Z)_{\tau}\right)\right)$.

(iv) If $K \subseteq C(Y, Z)$ is $\tau$-compact, then $K$ is $\tau_{p}$-closed in $F(Y, Z)$, pointwise bounded and e-continuous.

The assertion (i) $\Rightarrow$ (ii) above generalizes Theorem B of [44] (which is (ii) with $\left.\tau=\tau_{k}\right)$ since $\left(X, Y, Z, \tau_{k}\right)$ always satisfies the partial exponential law. The results of $\$ 2$ yield further conditions under which (ii) will hold.

The requirement that $K$ be $\tau_{p}$-closed in $F(Y, Z)$ is unpleasant, because it takes us outside of $C(Y, Z)$. We will show how this condition can be replaced by an "intrinsic" condition (which we are about to define) and the condition that $K$ be $\tau_{p}$-closed (in $C(Y, Z)$ ). This is still not altogether satisfactory, but we know of no way to avoid the requirement that $K$ be $\tau_{p}$-closed without assuming a property at least as strong as even continuity on a cover.

Let $S$ be a subset of $C(Y, Z)$. We call $S$ simply evenly continuous if for each $y_{0}$ in $Y$, each $z_{0}$ in $Z$, each neighborhood $U$ of $z_{0}$ and each ultrafilter $\phi$ on $S$, there exists a neighborhood $V$ of $y_{0}$ and a neighborhood $W$ of $z_{0}$ such that for each $y$ in $V$ there exists $H_{y}$ in $\phi$ such that $f(y)$ is in $U$ whenever $f$ is in $H_{y}$ and $f\left(y_{0}\right)$ is in $W$. In the sense that even continuity generalizes equicontinuity, simple even continuity generalizes the simple equicontinuity of [8], which is defined as follows: If $Z$ is completely regular Hausdorff, $S \subseteq C(Y, Z)$ is simply equicontinuous if for each $y_{0}$ in $Y$, each continuous pseudometric $d$ on $Z$, and each $\varepsilon>0$ and each ultrafilter $\phi$ on $S$, there exists a neighborhood $V$ of $y_{0}$ such that, for each $y$ in $V$ there exists $H_{y}$ in $\phi$ such that $d\left(f(y), f\left(y_{0}\right)\right)<\varepsilon$ for all $f$ in $H_{y}$.

5.3 Proposition. (i) If $Z$ is completely regular Hausdorff and $S \subseteq C(Y, Z)$ is simply equicontinuous, then $S$ is simply evenly continuous; 
(ii) If $Z$ is completely regular Hausdorff and $S \subseteq C(Y, Z)$ is pointwise bounded and simply evenly continuous, then $S$ is simply equicontinuous.

(iii) If $S \subseteq F(Y, Z)$ is simply evenly continuous, then $S \subseteq C(Y, Z)$.

(iv) If $K \subseteq C(Y, Z)$ is $\tau_{p}$-compact, then $K$ is simply evenly continuous.

(v) If $Z$ is regular and $S \subseteq C(Y, Z)$ is simply evenly continuous and pointwise bounded, then so is the $\tau_{p}$-closure in $F(Y, Z)$ of $S$.

Proof. Parts (i) and (ii) follow by the obvious generalizations of the proofs of $[25,7.22]$ and $[25,7.23]$ respectively.

(iii) Given $f_{0} \in S, y_{0} \in Y$ and a neighborhood $U$ of $z_{0}=f_{0}\left(y_{0}\right)$, let $\phi$ be the ultrafilter on $S$ with $\left\{f_{0}\right\} \in \phi$ and note that, by simple even continuity there exists a neighborhood $V$ of $y_{0}$ such that $f_{0}(V) \subseteq U$.

(iv) If $K$ is compact and $\phi$ is an ultrafilter on $K$, then there exists a point $f_{0}$ in $K$ with $\left\{f_{0}\right\} \in \phi$. Take $W=U, V=f_{0}^{-1}(U)$ and for $y \in V, H_{y}=\left\{f_{0}\right\}$.

(v) By (iv) it suffices to show that the $\tau_{p}$-closure of $S$ in $F(Y, Z)$ is contained in $C(Y, Z)$. Let $f_{0}$ be a point in the $\tau_{p}$-closure of $S$, let $y_{0} \in Y$, and let $U$ be a closed neighborhood of $z_{0}=f_{0}\left(y_{0}\right)$. Let $\phi$ be an ultrafilter on $S$ which converges (in the obvious sense) to $f_{0}$ and let $V$ be a neighborhood of $y_{0}, W$ a neighborhood of $z_{0}$, such that for each $y$ in $V$ there exists on $H_{y}$ in $\phi$ such that $f(y) \in U$ whenever $f \in H_{y}$ and $f\left(y_{0}\right) \in W$. Since $\phi=\left\{H_{\lambda}\right\}$ converges to $f_{0}$, there exists a $\lambda_{0}$ such that for $\lambda>\lambda_{0}$, $f\left(y_{0}\right) \in W$ for each $f$ in $H_{\lambda}$. Thus for $y$ in $V$, and $f$ in $H_{y} \cap H_{\lambda 0}$ for $\lambda>\lambda_{0}, f(y)$ is in $U$. Hence, since $U$ is closed, $f_{0}(y)$ is in $U$, i.e., $f_{0}(V) \subseteq U$. Therefore $f_{0}$ is continuous.

Clearly each evenly continuous family of functions is simply evenly continuous, and [45, Example 6.1] shows that a compact, simply evenly continuous family with a $\tau_{p}$-dense $e$-continuous subfamily need not be evenly continuous, or even $e$ continuous. Of course an $e$-continuous family need not be simply evenly continuous-for $Y$ locally compact $C(Y, Z)$ is e-continuous but need not be closed in $F(Y, Z)$. The following result, an immediate consequence of 5.3 , is a slight generalization of [8, Theorem 4.7].

5.4 COROLlaRY. Let $Z$ be regular Hausdorff; a subset of $C(Y, Z)$ is $\tau_{p}$-compact if and only if it is $\tau_{p}$-closed, pointwise bounded and simply evenly continuous.

5.5 THEOREM. If $Z$ is regular, the statement of Theorem 5.2 holds with (ii) and (iv) replaced, respectively, by the following two conditions:

(ii') If $K \subseteq C(Y, Z)$ is $\tau_{p}$-closed, pointwise bounded, e-continuous and simply evenly continuous, then $K$ is $\tau$-compact.

(iv') If $K \subseteq C(Y, Z)$ is $\tau$-compact, then $K$ is $\tau_{p}$-closed, pointwise bounded, $e$ continuous and simply evenly continuous.

As Brace notes, conditions such as simple even continuity can also be used in conjunction with even continuity on a cover $\alpha$ to ensure that the closure in $C_{\alpha}(Y, Z)$ of a subset of $C(Y, Z)$ will remain in $C(Y, Z)$. In particular, Brace's result, [8, Theorem 4.10] generalizes to yield: 
5.6 TheOREM. Let $Z$ be regular Hausdorff. A subset of $C(Y, Z)$ is $\tau_{k}$-compact if and only if it is $\tau_{k}$-closed, pointwise bounded, evenly continuous on compacta, and simply evenly continuous.

We now turn to theorems of Ascoli type which characterize or describe sets having properties other than compactness. (By way of historical motivation, note that Ascoli's original theorem, as well as the classical result given in [25, Problem 7-D] - which we generalize with Corollaries 5.10 and 5.11-both describe sequentially compact sets rather than compact sets.) Where $P$ denotes a topological property, we will need the following curious condition: If $X$ has $P$ and $Y$ is indiscrete, then $X \times Y$ has $P$. We call a property satisfying this condition "nice." While the common separation properties are not nice, most generalized compactness properties are. In particular, sequential compactness, countable compactness, paracompactness and the Lindelöf property are all nice. Recall that a topological property $P$ is called closed hereditary if each closed subspace of a space having $P$ also has $P$.

5.7 THEOREM. Let $Y$ be arbitrary, let $Z$ be regular Hausdorff and let $P$ be a topological property which is closed hereditary. Further, suppose there exists a cardinal $\boldsymbol{\aleph}$ such that $Y$ contains a dense subset of cardinality $\boldsymbol{\aleph}$ and each $\boldsymbol{\aleph}$-fold product of closed subspaces of $Z$ has $P$ whenever each of its factors has $P$. If $P$ is nice or card $(Y) \leqq \aleph$, and if $\tau \geqq \tau_{p}$ is a topology such that for each regular Hausdorff space $X$ having $P,(X, Y, Z, \tau)$ satisfies the partial exponential law, then a subspace $F$ of $C(Y, Z)_{\tau}$ has $P$ if it satisfies the following three conditions:

(i) $F$ is $\tau$-closed.

(ii) $F$ is evenly continuous.

(iii) For each $y$ in $Y, \operatorname{cl}\{f(y): f \in F\}$ has $P$.

Proof. Let $D \subseteq Y$ be a dense subset of cardinality $\aleph$, and suppose $F \subseteq C(Y, Z)$ satisfies the three conditions above. Let $\bar{F}$ denote the $\tau_{p}$-closure of $F$; $\bar{F}$ is evenly continuous so $\bar{F} \subseteq C(Y, Z)$ and, on $\bar{F}$, the topology of pointwise convergence on members of $D$ coincides with $\tau_{p}$. Also, where $F_{y}$ denotes the closure of $\{f(y): f \in F\}$, $\pi_{y \in D} F_{y}$ has $P$ and consequently, since $P$ is nice, $\bar{F}$ has $P$. Thus $(\bar{F}, Y, Z, \tau)$ satisfies the partial exponential law, so $\mu e: \bar{F} \rightarrow C(Y, Z)_{\tau}$ is continuous and is therefore a homeomorphism. But then the $\tau$-closed subspace, $F$, of $\bar{F}$ must have $P$.

For completeness we give as our next result the partial converse of the theorem above which the first portion of the proof of Theorem 1.2 easily generalizes to yield. Here the conditions on $P$ are quite stringent, but they do not force it to be compactness. For instance, if $P$ is sequential or countable compactness, if $Z$ is first countable (regular Hausdorff) and if $Y$ is countable and sequential, then the hypotheses are satisfied.

5.8 TheOREM. Let $Y$ be fixed, $Z$ be regular Hasudorff, let $\tau \geqq \tau_{p}$ and let $P$ be a topological property which is closed hereditary and such that if $X$ is any regular Hausdorff space which has $P$, then 
(i) $\pi_{Y}: X \times Y \rightarrow Y$ is closed (or, if $Z$ is completely regular, $z$-closed); and

(ii) If $g: X \rightarrow C(Y, Z)_{\tau_{p}}$ is one-to-one and continuous, then $g$ is a homeomorphism. If $C(Y, Z)_{\tau}$ satisfies the conclusion of Theorem 5.7 , then for each regular Hausdorff space having $P,(X, Y, Z, \tau)$ satisfies the partial exponential law.

Complementing Theorems 5.7 and 5.8, the proof of Theorem 1.3 generalizes to yield the following result:

5.9 Theorem. Let $Y$ be arbitrary, let $Z$ be regular, let $\tau \geqq \tau_{p}$ and let $P$ be a topological property which is preserved under continuous images (in $C(Y, Z)_{\tau}$ ). Then the following two conditions are equivalent:

(i) For each space $X$ having $P, C(X \times Y, Z) \subseteq \mu^{-1}\left(C\left(X, C(Y, Z)_{\tau}\right)\right)$.

(ii) Each subspace of $C(Y, Z)_{\tau}$ which has $P$ is evenly continuous.

5.10 Corollary. If $P, Y, Z$, and $\tau$ satisfy one of the conditions (i), (ii), etc., below, then a $\tau$-closed evenly continuous subspace $F$ of $C(Y, Z)_{\tau}$ has $P$ if for each $y$ in $Y, \mathrm{cl}\{f(y): f \in F\}$ has $P$. If $P, Y, Z$, and $\tau$ satisfy one of the conditions (i'), (iv') below, then each subspace of $C(Y, Z)_{\tau}$ which has $P$ is evenly continuous.

(i) $P=$ sequential compactness, $Y$ is separable, $Z$ is regular Hausdorff and $\tau_{p} \leqq \tau \leqq \tau_{k}$.

(i') $P=$ sequential compactness or countable compactness, $Y$ is a sequential space, $Z$ is regular and $\tau \geqq \tau_{s}$.

(ii) $P=$ countable compactness, $Y$ is separable, $Z$ is a Hausdorff $k$-space and either $Z$ is regular and $\tau_{p} \leqq \tau \leqq \tau_{k}$ or $Z$ is completely regular and $\tau_{p} \leqq \tau \leqq \tau_{\beta}^{\prime \prime}$.

(iii) $P=$ countable compactness, $Y$ contains a dense subset of cardinality at most $\aleph_{1}, Z$ is first countable Hausdorff and either $Z$ is regular and $\tau_{p} \leqq \tau \leqq \tau_{k}$ or $Z$ is completely regular and $\tau_{p} \leqq \tau \leqq \tau_{\beta}^{\prime \prime}$.

(iv) $P=$ Lindelöf, $Y$ is separable, $Z$ is regular Hausdorff and either $\sigma$-compact, or cosmic (continuous image of a separable metric space) or Lindelöf and complete in the sense of Čech, and $\tau_{p} \leqq \tau \leqq \tau_{k}$.

(iv') $P=$ Lindelöf, $Y$ is $\boldsymbol{\aleph}_{0}$-discrete, $Z$ is regular and $\tau \geqq \tau_{\alpha}$ for some cover which $\mathscr{S}_{0}$-generates $Y$. (Unless $Y$ is discrete, $\tau$ cannot be less than or equal to $\tau_{k}$.)

(v) $P=$ paracompactness, $Y$ is separable, $Z$ is metrizable and $\tau_{p} \leqq \tau \leqq \tau_{k}$.

Proof. Parts (i') and (iv') follow by 5.9 and 3.5 (parts (ii) and (v) respectively). Parts (ii) and (iii) follow by 5.7, 2.6 and the Remark following 2.6, and the appropriate product theorems, which appear in [32] and [40] respectively. Finally, parts (i), (iv) and (v) follow by 5.7, 2.8, and the appropriate product theorems; for (i) and (v) these are well known, and for (iv) these appear in [21] and [25].

For a more general condition under which (v) above will hold, see [34]. To an extent the theorem above, combined with an Ascoli theorem, yields conditions under which, for instance, each closed sequentially compact subspace of $C(Y, Z)_{\tau}$ is compact. For much stronger results along these lines, see [19]. Finally, let us note that our results can seldom be applied to show that all of $C(Y, Z)_{\tau}$ is compact, 
or countably compact, etc. For results in this direction, see [39] (compactness, countable compactness and paracompactness) and [13] (the Lindelöf property).

\section{REFERENCES}

1. R. Arens, A topology for spaces of transformations, Ann. of Math. (2) 47 (1946), 480-495.

2. R. Arens and J. Dugundji, Topologies for function spaces, Pacific J. Math. 1 (1951), 5-31.

3. C. Arzelà, Sull' integrabilita della equazioni differenziali ordinarie, Mem. Accad. Bologna (5) 5 (1895-1896), 257-270.

4. G. Ascoli, Le curve limite di una varietà data di curve, Mem. Accad. Lincei (3) 18 (1883), 521-586.

5. R. W. Bagley and J. S. Yang, On k-spaces and function spaces, Proc. Amer. Math. Soc. 17 (1966), 703-705.

6. R. G. Bartle, On compactness in functional analysis, Trans. Amer. Math. Soc. 59 (1955), 37-57.

7. J. W. Brace, The topology of almost uniform convergence, Pacific J. Math. 9 (1959), 643-652.

8. - Convergence on filters and simple equicontinuity, Illinois J. Math. 9 (1965), 286-296.

9. R. Brown, Function spaces and product topologies, Quart. J. Math. Oxford Ser. (2) 15 (1964), 238-250.

10. S. S. L. Chang, An extension of Ascoli's theorem and its applications to the theory of optimal control, Trans. Amer. Math. Soc. 120 (1965), 445-470.

11. D. E. Cohen, Spaces with weak topology, Quart. J. Math. Oxford Ser. (2) 5 (1954), $77-80$.

12. C. H. Cook and H. R. Fischer, On equicontinuity and continuous convergence, Math. Ann. 159 (1965), 94-104.

13. H. H. Corson and J. Lindenstrauss, On function spaces which are Lindelöf spaces, Trans. Amer. Math. Soc. 121 (1966), 479-491.

14. I. Fleischer and S. P. Franklin, On compactness and projections, (to appear).

15. R. H. Fox, On topologies for function spaces, Bull. Amer. Math. Soc. 51 (1945), 429-432.

16. A. Frölicher and W. Bucher, Calculus in vector spaces without norm, Springer-Verlag, New York, 1966.

17. Z. Frolik, The topological product of two pseudocompact spaces, Czechoslovak Math. J. (85) 10 (1960), 339-349.

18. D. Gale, Compact sets of functions and function rings, Proc. Amer. Math. Soc. 1 (1950), 303-308.

19. I. Glicksberg, Representation of functionals by integrals, Duke Math. J. 19 (1952), 253-282.

20. A. Grothendieck, Criteres de compacité dans les espaces fonctionnels generaux, Amer. J. Math. 74 (1952), 168-186.

21. A. W. Hager, Projections in zero sets (and the fine uniformity on a product), Trans. Amer. Math. Soc. 140 (1969), 87-94.

22. - Real continuous functions on a Cartesian product, (to appear).

23. J. R. Isbell, Uniform spaces, Math. Surveys No. 12, Amer. Math. Soc., Providence, R. I., 1964.

24. J. R. Jackson, Spaces of mappings on topological products with applications to homotopy theory, Proc. Amer. Math. Soc. 3 (1952), 327-333.

25. J. L. Kelley, General topology, Van Nostrand, New York, 1955.

26. E. Michael, $\boldsymbol{\aleph}_{0}$-spaces, J. Math. and Mech. 15 (1966), 983-1002. 
27. E. Michael, Local compactness and Cartesian products of quotient maps and k-spaces, (to appear).

28. K. Morita, On spaces having the weak topologies with respect to a closed cover. I, II, Proc. Japan. Acad. 28 (1953), 537-543; 30 (1954), 711-717.

29. - Note on mapping spaces, Proc. Japan. Acad. 32 (1956), 671-675.

30. S. Mrówka, On function spaces, Fund. Math. 45 (1958), 273-282.

31. S. B. Myers, Equicontinuous sets of mappings, Ann. of Math. 47 (1946), 496-502.

32. N. Noble, $k$-spaces and some generalizations, Ph.D. dissertation, University of Rochester, Rochester, N. Y., 1967. (University Microfilms \#67-13,637.)

33. - Products with closed projections, Trans. Amer. Math. Soc. 140 (1969), 381-391.

34. —- Countably compact and pseudocompact products, Czechoslovak Math J. (to appear).

35. - The continuity of functions on Cartesian products, (to appear).

36. A. Okuyama, Some generalizations of metric spaces, the metrization theorems and product spaces, Sci. Rep. Tokyo Kyoiku Daigaku Sect. A 9 (1968), 60-78.

37. H. Poppe, Stetig Konvergenz und der Satz von Ascoli und Arzelà, Math. Nachr. 30 (1965), 87-122.

38. - Stetig Konvergenz und der Satz von Ascoli und Arzelà. II, Monatsb. Deutsch. Akad. Wiss. Berlin 4 (1966), 259-264.

39. —- Stetig Konvergenz und der Satz von Ascoli und Arzelà. III, IV, V, VI, Proc. Japan. Acad. 44 (1968), 234-242, 318-323.

40. - Zum Satz von Ascoli und Arzelà, Math. Ann. 171 (1967), 46-53.

41. E. Spanier, Quasi-topologies, Duke Math. J. 30 (1963), 1-14.

42. A. H. Stone, A note on paracompactness and normality of mapping spaces, Proc. Amer. Math. Soc. 14 (1963), 81-83.

43. A. H. Stone and C. T. Scarborough, Products of nearly compact spaces, Trans. Amer. Math. Soc. 124 (1966), 131-147.

44. J. D. Weston, A generalization of Ascoli's theorem, Mathematika 6 (1959), 19-24.

45. J. S. Yang, Equicontinuity in function spaces, Ph.D. dissertation, University of Miami, Coral Gables, Fla., 1967. (University Microfilms \#67-9235.)

\section{Clark University,} Worcester, MassachusetTS 